\begin{tabular}{|l|c|c|c|c|c|}
\hline Cuadernos I. Geográfica & 16 & $1-2$ & $109-122$ & Logroño & 1990 \\
\hline
\end{tabular}

\title{
INCISIONES (RILLS) EN CAMPOS ABANDONADOS: PRIMERAS OBSERVACIONES SOBRE CAPACIDAD DE TRANSPORTE DE SEDIMENTOS
}

\author{
P. RUIZ-FLAÑO* \\ J. M. GARCIA-RUIZ*
}

\begin{abstract}
RESUMEN.-Se estudian las caracteristicas de las redes de incisiones en campos abandonados, en particular su capacidad para transportar sedimentos gruesos. Por medio de sencillas trampas de materiales en varias incisiones, se ha comprobado que en pocas ocasiones se produce movtlización de pledras y que, en todo caso, la proporción de estas últimas en el balance de sedimentos es muy pequeña. Las caracteristicas morfométricas de las incisiones demuestran que son sistemas muy inmaduros y poco organizados.
\end{abstract}

ABSTRACT.-Rills in abandoned fields: firstobservations about their capacity to carry sediments. The characteristics of the rill networks in abandoned fields are studied, mainly their capacity to carry coarse sediments. Using single sediment traps in several rills, we prove that only in few occasions stones move and that, in all cases, their proportion in the sediment balance is very small. The morphometric features of the rills show that they are quite inmature, little organized systems.

Palabras clave: Incisiones, piedras, transporte, tormentas, campos abandonados.

Key words: Rills, stones, sediment transport, rainstorms, abandoned fields.

Los campos abandonados de las regiones de montaña soportan una amplia gama de procesos geomórficos, que varian en el tiempo y en el espacio. Esta diversidad se explica básicamente por la complejidad de la sucesión vegetal, por el efecto de los usos del suelo anteriores y posteriores al abandono -con

*Instituto Prenaico de Ecologia, C.S.I.C., Campus de Aula Del. Carretera de Montañana, s/n, Apartado 202, 50080-Zaragoza. 
influencia directa en el comportamiento hidrológico de los suelos-, por el papel de diversos factores topográficos (pendiente y forma de la ladera, sobre todo) y por la diferente edad de abandono. En este último caso, RuIZ-FLAÑo et al (en prensa) comprobaron que con el paso del tiempo se produce un cambio importante en los procesos geomórficos dominantes y en el aspecto y capacidad productiva de los campos abandonados. Así, mientras en los campos abandonados recientemente predomina la erosión difusa, debido a las características de la cubierta vegetal, con el paso del tiempo aparecen pequeñas acumulaciones vinculadas a pies de matorral, incisiones, coladas de piedras y pavimento de piedras. Los campos más antiguos muestran una imagen deteriorada, con mucha pedregosidad y un matorral submediterráneo abierto que sustenta manchas aisladas de suelo en fase de avanzada degradación.

En cualquier ladera, uno de los problemas básicos es averiguar la capacidad de producción de sedimentos y la eficacia de la escorrentía superficial para transportar materiales de diferente calibre. Esta eficacia depende de las características de la cubierta vegetal y del suelo, de la intensidad de las precipitaciones y del modo en que fluyen las aguas. Cuando la capacidad de infiltración se halla muy limitada, la escorrentía superficial tiende a concentrarse en pequeños canalillos (rills, incisiones, rigolas), que aumentan la energia disponible para exportar sedimentos en varios órdenes de magnitud (POESEN, 1987). El conocimiento de la evolución y dinámica de estas pequeñas redes de drenaje es, pues, fundamental para interpretar la intensidad de las pérdidas de suelo y la organización espacial de los procesos geomórficos, de la pedregosidad y, parcialmente, de la vegetación. En este trabajo se estudian algunas características morfológicas de los canalillos en campos abandonados y se relacionan con su capacidad para evacuar sedimentos en diferentes eventos pluviométricos.

\section{Area de estudio}

El estudio se centra en los campos abandonados en pendiente situados en las laderas del flysch, que litológica y geomorfológicamente presentan una gran homogeneidad. El muestreo se ha llevado a cabo en el valle de Aisa (Fig.1), drenado por el río Estarrún. El relieve se caracteriza por una serie de aineaciones que descienden de Norte a Sur, con divisorias alomadas y laderas regularizadas, afectadas por movimientos en masa de diferente entidad $y$ procesos vinculados al arroyamiento superficial.

El clima es mediterráneo de montaña, ligeramente continentalizado. Las precipitaciones (entre 800 y $2.000 \mathrm{~mm}$.) se concentran en primavera e invierno, acusándose un ligero déficit estival en las laderas más bajas. Las umbrias se encuentran dominadas por bosques de Pinus sylvestris, mientras que las solanas están ocupadas por campos abandonados de pequeño tamaño $(<3.000$ $\mathrm{m}^{2}$ ) alternando con bosquetes de Quercus gr. faginea. 
Los suelos de los antiguos campos de cultivo son pardos, muy pedregosos y de escasa potencia. Son pobres en nutrientes y materia orgánica (1.5\%) y ricos en carbonatos. Su pH oscila entre 8 y 8.5 .

Pastoreo de ovino y vacuno en las estaciones intermedias y repoblaciones forestales constituyen el uso actual de estos espacios, que hasta el momento de su abandono se dedicaban al cultivo de cereales alternando con barbechos más o menos prolongados.

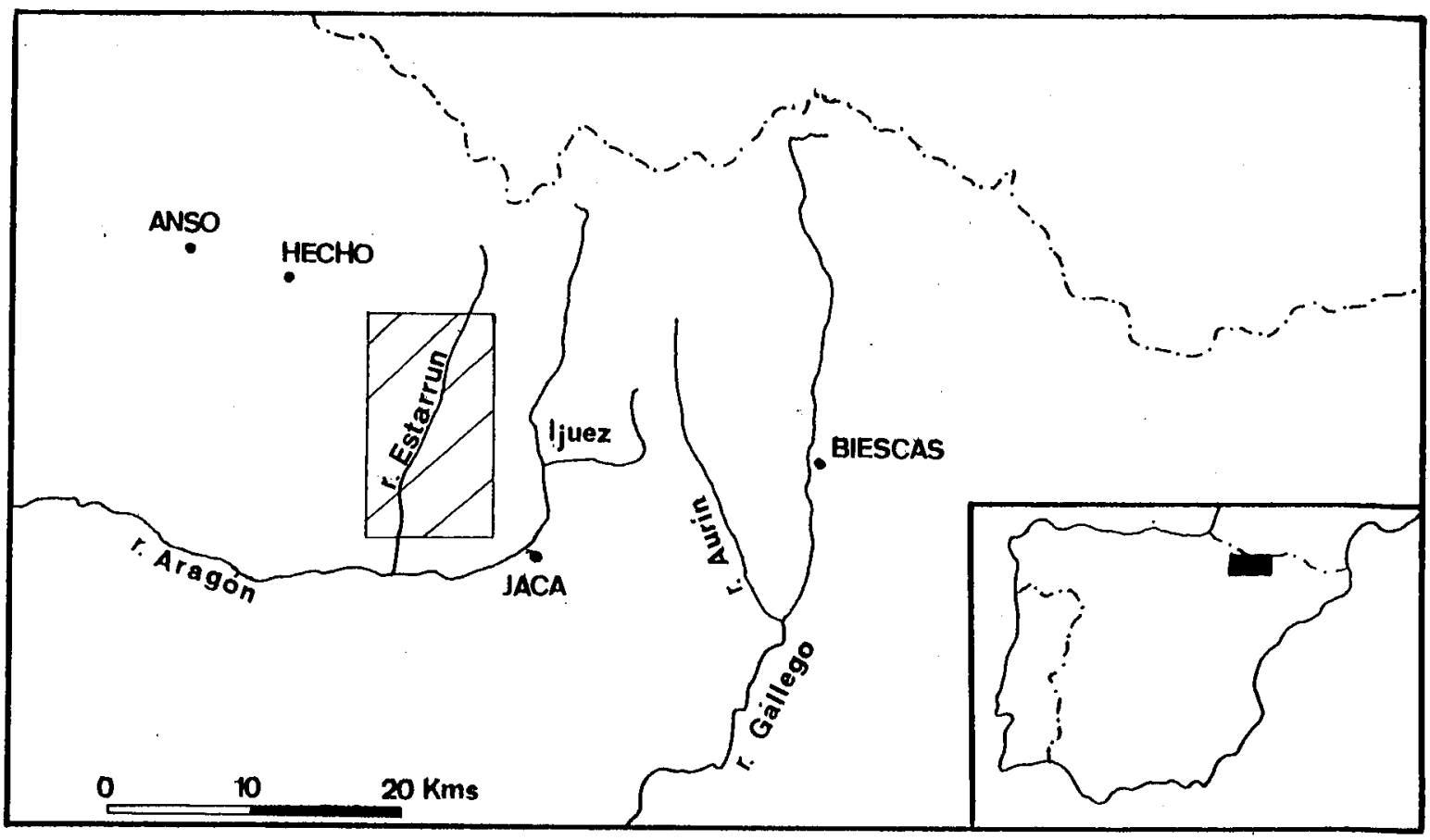

Flg.1. Area de Estudio

\section{Métodos}

Se han seleccionado tres parcelas cuyas edades oscilan entre 25 y 100 años de abandono aproximadamente. En cada una de ellas se ha realizado una cartografia geomorfológica de detalle, a escala 1:125, que incluye el trazado minucioso de la red de drenaje y un exhaustivo muestreo de la pendiente. Se ha procedido a medir la anchura y profundidad de cada una de las incisiones desde la parte superior a la parte inferior de las mismas, así como el tamaño de las piedras a lo largo de todo su recorrido.

En una de las parcelas se han construído 3 pequeñas trampas de sedimentos que han sido posteriormente recubiertas con una malla de $2 \mathrm{~mm}$. de luz (Foto 2), con el fin de retener el sedimento grueso movilizado por las incisiones en cada precipitación. Para determinar la cantidad de sedimento fino exportado por cada rigola en los mismo eventos, disponemos de la información procedente de pequeñas parcelas de $3 \mathrm{~m}^{2}$ de superficie aproximada, cerradas con cajas 


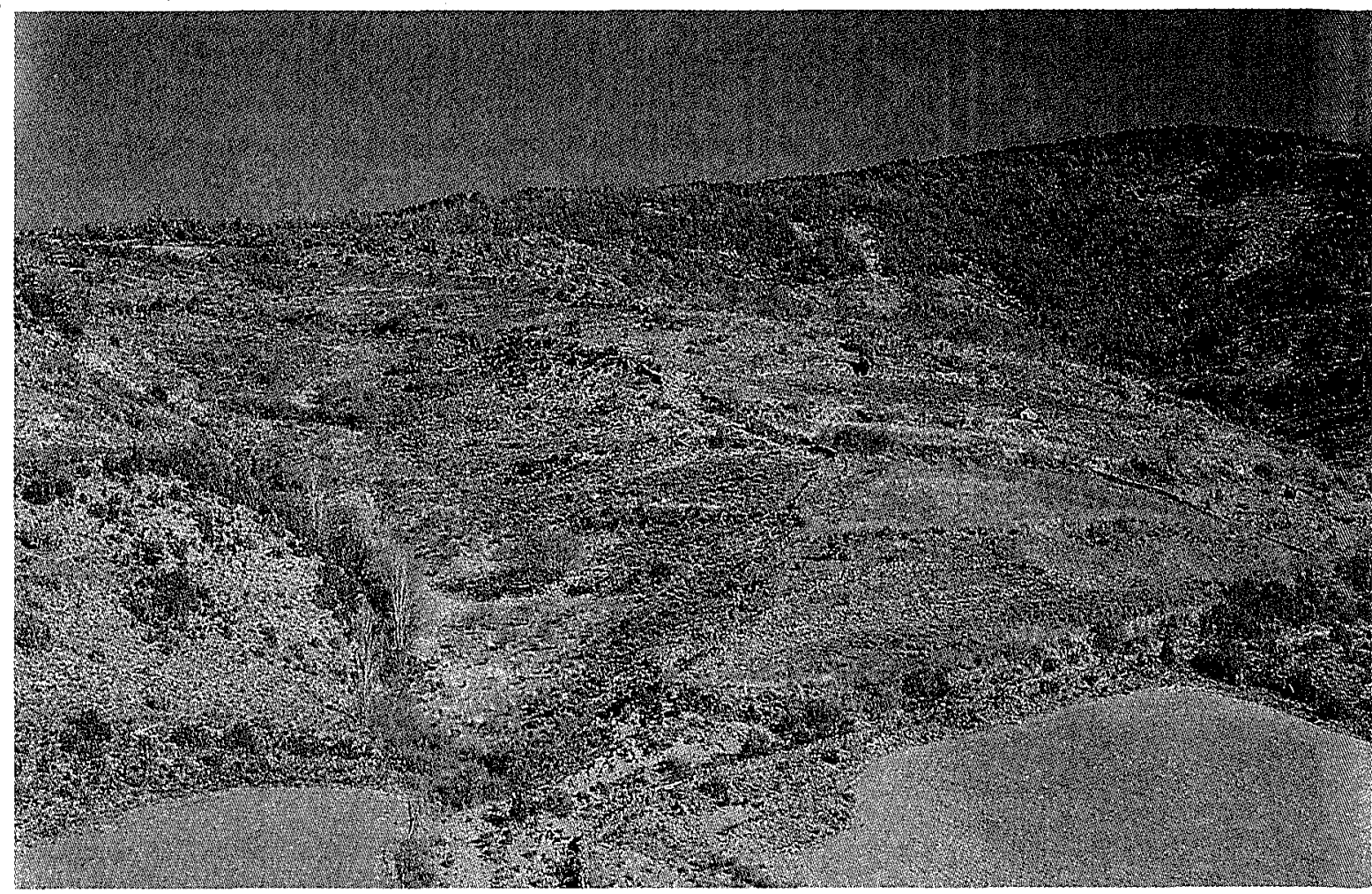

Foto 1. Iadera de campos abandonados en el valle de Aisa.

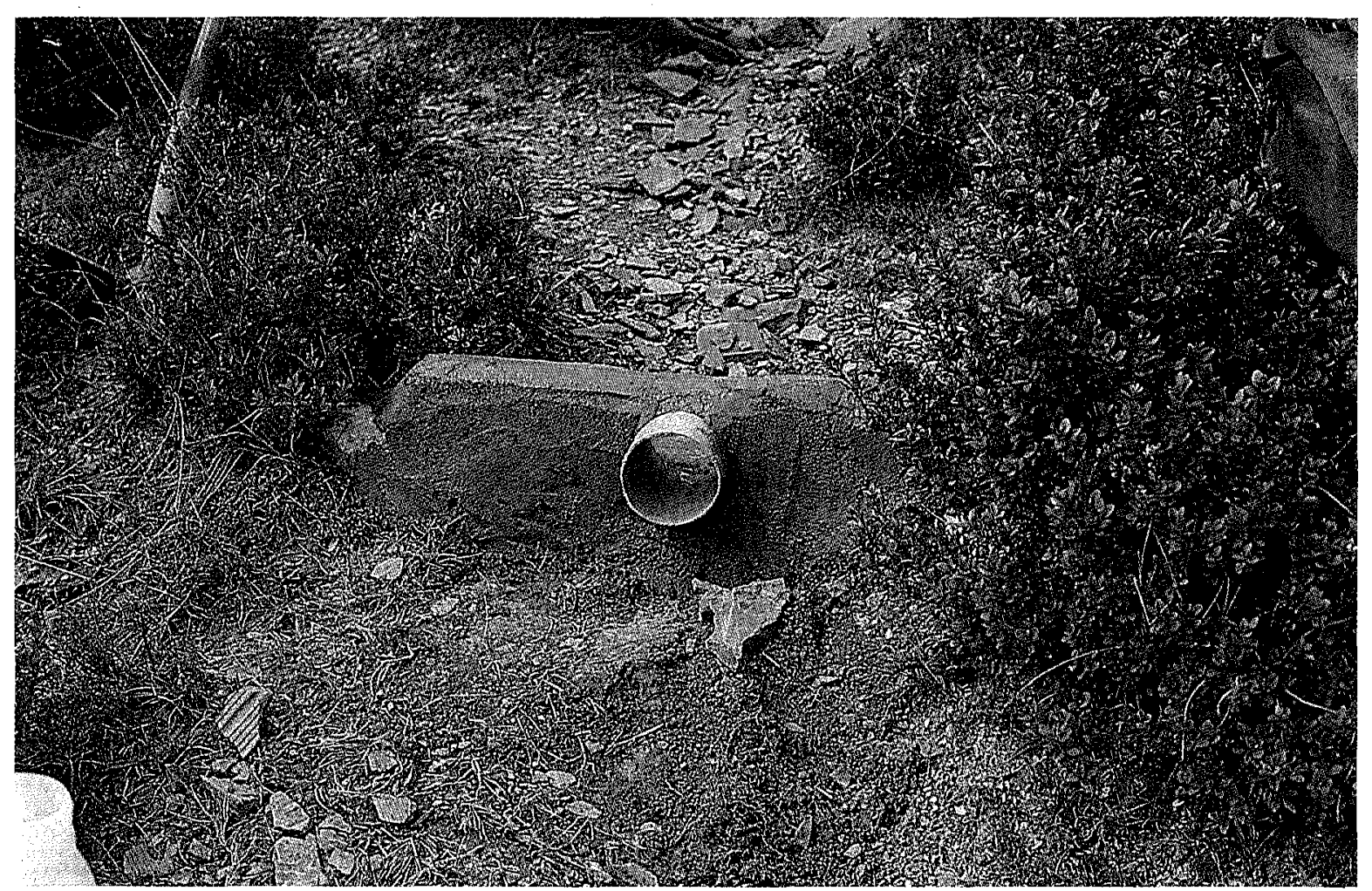

Foto 2. Pequeña trampa de sedimentos en Incisıón formada en un campo abandonado hace más de 50 años. Sinués, valle de Aísa. 
Gerlach. La información hidrológica y sedimentológica procedente de estas parcelas, ubicadas en ambientes muy degradados próximos a las trampas, ha sido extrapolada a la superficie total drenada por cada una de las 3 incisiones, por lo que este dato ha de ser tomado como una aproximación, y, en todo caso, el dato manejado constituye un umbral minimo, que se utiliza para disponer de un orden de magnitud sobre la exportación de sedimentos en suspensión.

En otra de las parcelas se pintaron piedras de diferente tamaño, localizadas tanto en incisiones como en espacios interrills, para controlar la movilidad y la longitud de sus desplazamientos en tormentas individuales. Finalmente disponemos de información sobre la cuantia e intensidad de las distintas precipitaciones.

\section{Resultados}

La tabla 1 incluye información sobre la red de incisiones de las tres parcelas seleccionadas. La mayor densidad de drenaje se da en la parcela de menor edad, aunque en ella la profundidad media de las incisiones es mucho más reducida. Con una densidad menor aparece la parcela con más de 80 años de abandono y, mucho más alejada, la de edad de abandono intermedio. Esta última posee sólo tres canales que concentran rápidamente todas las aguas de escorrentía de la parcela sin dar lugar a ramificaciones importantes de la red de drenaje dada la elevada pendiente de la misma, que en algunos tramos supera el $40-50 \%$.

TABLA 1

Caracterización de las redes de incisiones en tres parcelas de diferente edad.

\begin{tabular}{cccccc}
\hline Parcela & Edad & $\begin{array}{c}\text { Long. incis. } \\
(\mathrm{m})\end{array}$ & $\begin{array}{c}\text { Anch. incis. } \\
(\mathrm{mm})\end{array}$ & $\begin{array}{c}\text { Prof. incis } \\
(\mathrm{mm})\end{array}$ & $\begin{array}{c}\text { Densidad de } \\
\text { incisiones (m/Ha) }\end{array}$ \\
\hline P1 & $25-50$ & 111 & 545 & 80 & 4259 \\
P2 & $>50$ & 41 & 816 & 72 & 455 \\
P3 & $>80$ & 190 & 311 & 38 & 2578 \\
\hline
\end{tabular}

Las figuras $2 \mathrm{~A}$ y $2 \mathrm{~B}$ corresponden a la cartografia detallada de las dos parcelas de mayor edad. Ambas se encuentran colonizadas por un matorral abierto y poseen numerosos pies de vegetación arbórea (Pinus sylvestris). La erosión difusa fuerte es el microambiente geomorfológico más extendido, sobre todo en la parte alta y media de la parcela, donde la pedregosidad superficial es más importante debido al lavado de finos efectuado por el arroyamiento no concentrado. Aguas arriba de los pies de matorral se producen acumulaciones de material heterométrico que, en ocasiones, dan lugar a un enlosado de 


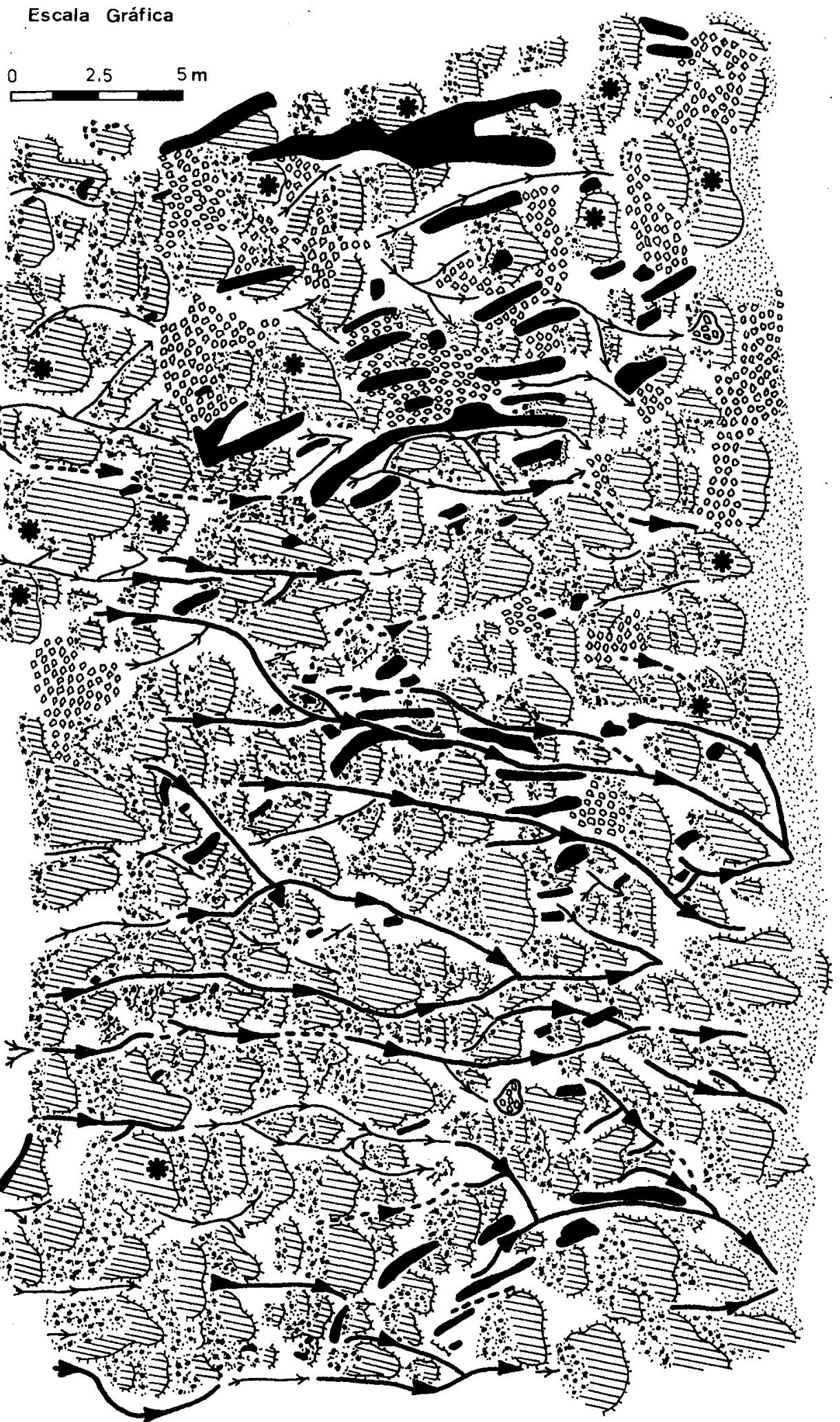

Fig. 2A. Esquema geomorfólógıco de una parcela abandonada hace mảs de 80 años en SInuẽs, valle de Aisa. Ver Leyenda. 
INCISIONES (RILLS) EN CAMPOS ABANDONADOS

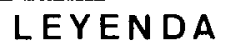




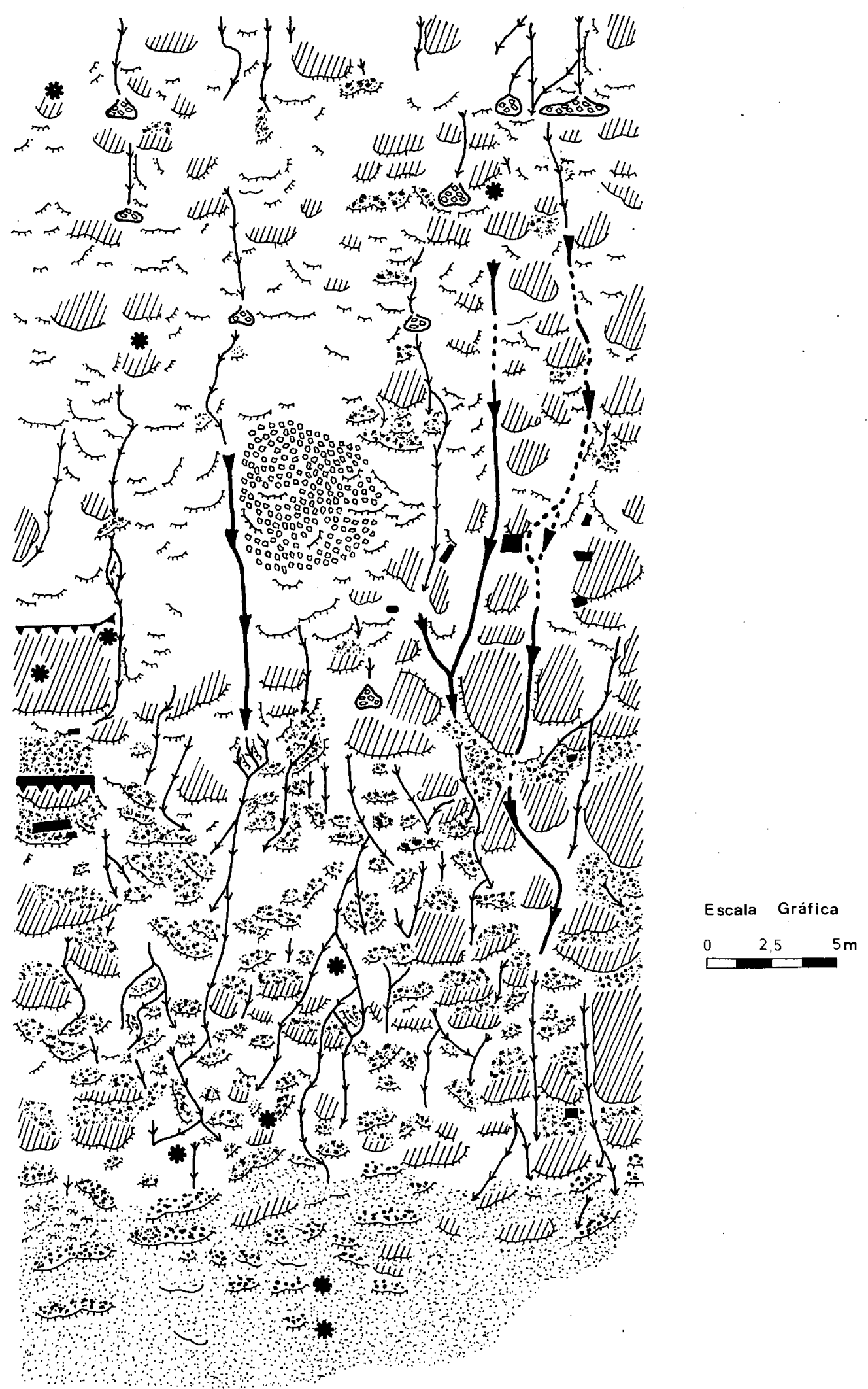

FIg. 2B. Esquema geomorflógico de una parcela abandonada hace 50 años en Aisa. 
piedras. En las zonas inmediatamente inferiores a dichos pies, el matorral se encuentra descalzado como consecuencia de la concentración del agua en sus bordes y por acción del mayor poder erosivo del agua concentrada en los tallos de la vegetación (stemflow) que produce un socavamiento de la base frontal del matorral. La pedregosidad superficial disminuye en la parte inferior de la parcela, donde la suavización de la pendiente da lugar a una importante deposición de material fino en el que ocasionalmente se pueden observar las huellas de la erosión difusa fuerte en la formación de pináculos.

En los esquemas (2A y B), las incisiones comienzan a una cierta distancia del borde superior del campo, una vez las aguas de escorrentia han alcanzado un cierto grado de velocidad y pendiente, de manera que el arroyamiento difuso pasa a concentrado (HORTON, 1945). Las cabeceras de las incisiones no presentan límites definidos, con una forma muy ligeramente cóncava, apenas apreciable, con elevada pedregosidad superficial. La densidad de drenaje en estos sectores superiores de la parcelas es todavia muy reducida, incrementándose en las zonas medias (asi, en la parcela $2 \mathrm{~A}$ se pasa de una densidad de 2.054 m.ha ${ }^{-1}$ a 2.727 m.ha ${ }^{-1}$; y en la parcela $2 \mathrm{~B}$, de 297 a $723 \mathrm{~m}$. ha ${ }^{-1}$ respectivamente).

En el tramo medio, las incisiones se hallan muy débilmente integradas y frecuentemente aprovechan los abundantes afloramientos de sustrato rocoso para encajarse en el contacto entre estrato duro y estrato blando. Una vez se ha iniciado el encajamiento de la rigola, éste no es continuo a lo largo de todo su recorrido: unas veces se pierde por completo la incisión y se recupera unos metros más abajo; otras, en cambio, es sustituida momentáneamente por coladas de piedras que, posteriormente, pasan a incisión. Incluso existen difluencias provocadas por la presencia de matorral que divide el flujo hídrico hacia los laterales. En la parte baja de la parcela son posibles dos alternativas: i) La incisión alcanza la parte inferior del campo y se encaja por efecto de la erosión remontante en el salto a otra parcela; ii) La incisión es sustituída por una colada de piedras con formación de conos de sedimentos gruesos que constituyen el depósito de materiales arrastrados por el canalillo. En cualquier caso, las coladas no suelen llegar a los últimos metros de la parcela, caracterizados por acumulación de material fino con una insignificante proporción de sedimento grueso.

Diversos autores (CARRAGHER et al, 1983; KLEIN, 1981; LEOPOLD \& MADDOCK, 1953) han demostrado que a medida que aumenta la superficie de la cuenca vertiente se produce un progresivo incremento de la dimensión de las incisiones. En el caso de los canalillos que nos ocupan, los resultados obtenidos de la medición de anchura y profundidad resultan aparentemente sorprendentes. Ninguna relación significativa ha sido obtenida entre la distancia desde el inicio del canal y los dos parámetros anteriores. Para cuencas pequeñas, CARRAGHER et al (1983) señalan la existencia de una clara relación entre anchura y área de drenaje y remarcan que pueden existir puntos de ruptura en estas relaciones que pueden atribuirse a los efectos del tamaño del material del lecho. Basándonos en esta afirmación, pusimos en relación un mayor número de parámetros para apreciar el grado de dependencia establecido entre todos ellos: anchura, 
profundidad, distancia, sección del canal, pendiente local, tamaño medio de las piedras del canal, tamaño medio de las tres piedras mayores, tamaño medio de las tres menores y porcentaje de finos en el canal. Sin embargo, a excepción de las aparentemente obvias, tampoco en este caso encontramos relaciones significativas. No hemos podido comprobar el efecto del tamaño del material del lecho y tan sólo una relación significativa y negativa $(r=-0.86)$ entre la distancia y el porcentaje de canal desprovisto de piedras en una de las rigolas merece la pena destacarse. La presencia masiva de piedras en los últimos tramos del canal probaria, al menos parcialmente, la existencia de una reducción de la capacidad de transporte hacia aguas abajo.

Es evidente que una cuestión muy relacionada con la actividad de las incisiones es la mayor o menor presencia de piedras en el lecho del canal. Algunas incisiones carecen de piedras en gran parte de su recorrido y muestran signos de ser muy activos (afloramientos de raices y del sustrato rocoso, por ejemplo). Otras, por el contrario, presentan gran pedregosidad y a simple vista parecen inactivas. Para comprobar la capacidad de movilización de piedras y la distancia a la que pueden desplazarse, pintamos cantos de diferentes tamaños en incisiones de la figura 2B. Desde julio de 1989 hasta junio de 1990 sólo se ha producido un desplazamiento significativo el dia 11 de agosto de 1989 , con una tormenta de intensidad igual a $12 \mathrm{l} / \mathrm{h}$. Los resultados muestran una gran variabilidad tanto en la longitud de los desplazamientos (entre 0 y $5 \mathrm{~m}$.) como en el tamaño de las piedras movilizadas, aunque, en general las piedras superiores a $70 \mathrm{~mm}$. permanecen en el lugar original. La consecuencia de esta tormenta fue la formación de acumulaciones pedregosas -en forma de coladas de piedras- al final de cada incisión como un almacén de sedimentos que no llegó a la parte más baja de la parcela. Después de esta precipitación se volvieron a controlar nuevas piedras mediante pintura, pero no se han registrado más desplazamientos, incluso en periodos de pluviosidad muy importante.

Finalmente, los datos obtenidos a partir de las trampas de sedimentos permiten valorar la capacidad de transporte de las incisiones por lo que respecta a los materiales gruesos. La tabla 2 muestra el transporte de sedimentos producidos en tres canalillos. Es importante tener en cuenta que entre el 6 de mayo y el 20 de junio de 1990 se registró un total de 20 dias con precipitación, pero sólo en las tres ocasiones que se señalan en la tabla 2 hubo deposición de materiales gruesos en la trampa de sedimentos, incluso aunque la cantidad de lluvia fue bastante apreciable en algunos casos y con el suelo prácticamente saturado. En las tres tormentas existe una clara relación entre la cantidad de sedimento grueso recogido y el área de la cuenca. Así, la incisión $\mathrm{n}^{\mathrm{0}} 3\left(508 \mathrm{~m}^{2}\right)$ aporta entre 2.4 y 4.6 veces más material que la $n^{2} 2\left(89 \mathrm{~m}^{2}\right)$ y ésta, a su vez, ente 1.4 y 1.7 veces más que la $\mathrm{n}^{\circ} 1\left(34 \mathrm{~m}^{2}\right)$. Considerando la concentración de sedimento en suspensión en microparcelas localizadas en ambientes geomorfológicos muy degradados (erosión difusa severa), los materiales gruesos disminuyen su proporción en el balance de sedimentos desde la primera a la tercera tormenta. En todo caso, la proporción de gruesos respecto a la de sedimentos en suspensión es siempre muy baja, inferior al 5\% -y aún al 1\%-en algunos casos. 
INCISIONES (RILLS) EN CAMPOS ABANDONADOS

TABLA 2

Transporte de sedimentos por tres incisiones en tres tormentas.

\begin{tabular}{|c|c|c|c|}
\hline & Mayo 10,1990 & Mayo 22, 1990 & Junio 20,1990 \\
\hline $\mathrm{P}(\mathrm{mm})$ & 17.5 & 71 & 34 \\
\hline Coef. escorrentia & 0.9 & 0.9 & 0.8 \\
\hline Concentr.Sed.Susp. & 8.2 & 11.6 & 3.7 \\
\hline Intens. Precip. (1/m) & 0.05 & 0.08 & 0.33 \\
\hline \multicolumn{4}{|l|}{ Incistón $1\left(34 \mathrm{~m}^{2}\right)$} \\
\hline Q. $\max \cdot \min ^{-1}(1)$ & 1.7 & 3.4 & 11.2 \\
\hline Lluvia total en la cuenca(1) & 595 & 2414 & 1156 \\
\hline Escorrentía (l) & 535 & 2173 & 925 \\
\hline Sed.Susp.(gr.) & 4391 & 25202 & 3421 \\
\hline Sed. Grueso(gr.) & 3807 & 4432 & 97 \\
\hline 2-4 mm(gr.) & 1184 & 1089 & 32 \\
\hline 4-8 mm(gr.) & 1473 & 1888 & 32 \\
\hline 8-30 mm(gr.) & 987 & 1081 & 33 \\
\hline + $30 \mathrm{~mm}$ (gr.) & 163 & 374 & - \\
\hline Total Sed.(gr.) & 8198 & 29634 & 3518 \\
\hline \%Sed. Susp. & 53.6 & 85 & 97.2 \\
\hline \%Sed. Grueso & 46.3 & 15 & 2.8 \\
\hline \multicolumn{4}{|l|}{ Incisión $2\left(89 \mathrm{~m}^{2}\right)$} \\
\hline Q. $\max \cdot \min ^{-1}(1)$ & 4.5 & 8.9 & 29.4 \\
\hline Lluvia total en la cuenca(l) & 1557 & 6319 & 3026 \\
\hline Escorrentia(1) & 1401 & 5687 & 2421 \\
\hline Sed.Susp.(gr.) & 11490 & 65970 & 8958 \\
\hline Sed.Grueso(gr.) & 6615 & 4750 & 137 \\
\hline 2-4 mm(gr.) & 1644 & 1585 & 61.3 \\
\hline 4-8 mm(gr.) & 2477 & 1800 & 58 \\
\hline 8-30 mm(gr.) & 2455 & 1289 & 18 \\
\hline +30 mm(gr.) & 39 & 76 & - \\
\hline Total Sed.(gr.) & 18105 & 70720 & 9095 \\
\hline \%Sed.Susp. & 63.5 & 93.3 & 98.5 \\
\hline \%Sed.Grueso & 36.5 & 6.7 & 1.5 \\
\hline \multicolumn{4}{|l|}{ Incisión $3\left(508 \mathrm{~m}^{2}\right)$} \\
\hline Q. $\max \cdot \min ^{-1}(1)$ & 25.4 & 50.8 & 167.6 \\
\hline Lluvia total en la cuenca(l) & 8890 & 36068 & 17272 \\
\hline Escorrentia(l) & 8001 & 32461 & 13818 \\
\hline Sed.Susp.(gr.) & 65608 & 376550 & 51123 \\
\hline Sed.Grueso(gr.) & 18765 & 21975 & 336 \\
\hline 2-4 mm(gr.) & 4943 & 5104 & 167 \\
\hline 4-8 mm(gr.) & 7670 & 7695 & 83 \\
\hline 8-30 mm(gr.) & 5056 & 8190 & 86 \\
\hline +30 mm(gr.) & 1096 & 986 & - \\
\hline Total Sed.(gr.) & 84373 & 398525 & 51459 \\
\hline \%Sed.Susp. & 77.8 & 94.5 & 99.3 \\
\hline \%Sed.Grueso & 22.2 & 5.5 & 0.7 \\
\hline
\end{tabular}


Otra cuestión importante es que, asî como el tamaño de la cuenca tiene gran importancia para explicar el volumen de gruesos registrado, no sucede lo mismo con el volumen de precipitaciones. Es evidente que debe existir un umbral por debajo del cual la lluvia carece de capacidad para producir escorrentia superficial con energia suficiente para arrastrar sedimentos gruesos. Pero por encima de ese umbral los resultados son más discutibles. Así, la lluvia del 20 de junio fue doble que la del dia 10 de mayo y, sin embargo, la cantidad de sedimentos gruesos transportados fue sensiblemente menor. Este problema requiere, no obstante, un número de observaciones muy superior al disponible hasta la fecha.

\section{Discusión y conclusiones}

Aunque anteriores estudios (RuIZ-FLAÑo et al, en prensa; RuIz-Flaño et al, 1990) nos han permitido valorar la diversidad de microambientes geomorfológicos instalados en campos abandonados en pendiente, es todavía escasa la información que poseemos sobre el origen y evolución de las incisiones en estas zonas. El arroyamiento difuso, primer proceso que se instala en el campo tras su abandono, se ve seguido en el tiempo de una organización espacial mucho más compleja. El resultado es el progresivo deterioro de la capacidad productiva del territorio y de su calidad paisajistica, proceso que alcanza una de sus más importantes expresiones con la aparición de incisiones. Una pequeña proporción superficial de este microambiente sugiere un avanzado estado de deterioro geomorfológico de la parcela, y aunque no conocemos bien los mecanismos a los que responde la instalación de las redes de drenaje, éstas incrementan su presencia con la edad. En una anterior publicación hemos sugerido la relación existente entre el uso ganadero del territorio, con fuegos periódicos que ayudan a mejorar temporalmente la calidad del pasto interrumpiendo la sucesión vegetal, y la formación de rigolas. Es evidente, por otro lado, que toda red de drenaje necesita tiempo y condiciones favorables para instalarse y el fuego puede ser una de estas condiciones ya que promueve la escorrentía superficial, tanto difusa como concentrada.

La información disponible sugiere las siguientes consideraciones sobre la evolución y capacidad de transporte de las incisiones:

a) Cuando el deterioro geomorfológico de la parcela abandonada está muy avanzado, las aguas de escorrentía tienden a concentrarse en pocos canales pero con una mayor competencia de arrastre, lo que responde a la necesidad de una mayor capacidad de evacuación de agua y sedimentos. Ello explica que la parcela de menor edad posea una mayor densidad de canalillos, pero que éstos tengan una reducida sección.

b) Las redes de drenaje con canales de mayor sección se instalan en los campos abandonados más antiguos, geomorfológicamente muy deteriorados, con estructura de matorral abierto y elevada pedregosidad superficial. Las 
mayores densidades de drenaje se alcanzan en la zona media de la parcela, donde se produce un incremento de la pendiente y donde la escorrentía superficial posee suficiente volumen y energia como para incrementar su capacidad erosiva. Se trata, no obstante, de un sistema inmaduro, débilmente jerarquizado y poco organizado a pesar del tiempo transcurrido desde el abandono (más de 80 años). Las dimensiones de los canales no se relacionan con la superficie de la cuenca y son muy pocos los que logran llegar a la parte inferior de la parcela. Esta escasa organización debe atribuirse, sobre todo a la elevada pedregosidad superficial y a la presencia de pies de matorral, que disipan la energia de las aguas de lluvia y de escorrentía. Experimentos llevados a cabo por POESEN et al (1990) demuestran que la elevada pedregosidad reduce el transporte de sedimentos y dificulta notablemente las posibilidades de concentración de la escorrentia superficial. La intensidad de las precipitaciones debe ser otro de los factores limitantes, pues en el área de estudio las lluvias no alcanzan ni la intensidad de otras áreas más mediterráneas al Este ni la duración y volumen de los ambientes más oceánicos a occidente. Por último, no debe olvidarse que las parcelas estudiadas se localizan sobre sustrato flysch, que localmente incluso aflora en superficie dentro del campo; la presencia de estratos duros de arenisca dificulta, sin duda, la progresión de las incisiones.

c) Las incisiones de campos abandonados constituyen sistemas muy limitados para movilizar materiales gruesos como demuestran los datos disponibles sobre el control de sedimentos en las tres incisiones y el abrumador predominio del sedimento en suspensión sobre el balance total. La intensidad de las precipitaciones y la disponibilidad de sedimento grueso en el lecho parecen ser los factores que controlan la evacuación de sedimentos. Los resultados incluídos en la tabla 2 demuestran que después de uno o varios eventos pluviométricos importantes, la disponibilidad de sedimentos gruesos en la incisión se agota, y que es necesario un largo lapso de tiempo, con tormentas menores, para que de nuevo accedan materiales al canal, en un fenómeno muy similar al que se produce en ríos más importantes (HAYWARD, 1980; MARTINEZ-CASTROVIEJO \& GARCÍA-RUIZ, en prensa).

d) En los espacios interrills, la erosión difusa fuerte es el proceso más extendido, con lavado de finos e incremento de la pedregosidad superficial. De acuerdo con POESEN (1987), en los espacios interrills la capacidad de transporte de materiales gruesos es más reducida que en los canalillos, y de hecho, ninguna piedra ha sido arrastrada hacia las cajas Gerlach. Sin embargo, el transporte de gruesos por erosión difusa severa debe ser ocasionalmente posible, como lo prueba la presencia de cantos en acumulaciones sedimentarias localizadas aguas arriba de pies de matorral.

Las parcelas abandonadas se colonizan pronto con una densa cubierta de herbáceas y de matorral, aunque la gestión ganadera puede fomentar la exportación de sedimentos finos por procesos de escorrentía difusa. La instalación de incisiones lleva normalmente bastante tiempo, de manera que sólo las encontramos en los campos de abandono más antiguo. Es evidente que la pedregosidad y el matorral constituyen frenos importantes para la progresión 
de canales, que en todo caso muestran una notoria incapacidad para evacuar volúmenes importantes de sedimentos gruesos incluso aunque las condiciones sean favorables. Es la exportación continua de sedimentos finos lo que explica la elevada pedregosidad superficial, que precisamente bloquea la progresión de las incisiones y limita en última instancia la energia disponible para transportar materiales más gruesos.

Agradecimientos. Este trabajo se ha realizado dentro del proyecto "Erosión en campos abandonados del Pirineo Central", integrado en el programa LUCDEME (Lucha contra la Desertización en el Mediterráneo) y dentro del proyecto "Erosión y desertización inducidos por el abandono de explotaciones agricolas en montaña”, financiado por la Comisión Interministerial de Investigación Científica y Técnica (CICYT).

\section{Bibliografia}

CARRAGHeR, M.J., KLEIN, M. \& PETCH, J.R., 1983.- Channel width-drainage area relations in small basins. Earth Surface Processes, 8: 177-181.

DE PlOEY, J., 1981.- The ambivalent effects of some factors of erosion. Mém. Ins. Géol. Univ. Louvain, 7, XXXI, pp. 171-181.

HAYWARD, J.A., 1980.- Hydrology and stream sediment from Torlesse stream catchment. Tussock Grasslands-Mountain Lands Institute, 236 pp., Canterbury, N.Z.

HorTon, R.E., 1945.- Erosional development of streams and their drainage basins: hydrophysical approach to quantitative geomorphology. Geological Society of America Bulletin, 56: 275-370.

KLEIN, M., 1981.- Drainage area and the variation of channel geometry downstream. Earth Surface Processes, 6: 589-593.

LEOPOLD, L.B. \& MADDOCK, T., 1953.- The hydralic geometry of stream channels and some physiographic implications. U.S. Geological Survey Prof. Paper, 252.

MARTÍNEZ-CASTROVIEJO, R. \& GARCIA-RUIZ,J.M., en prensa.- Coarse sediment transport in an experimental high mountain catchment of Central Pyrenees, Spain. Zeitschrift $f$. Geomorphologie, Suppl. Bd.

POESEN, J., 1987.- Transport of rock fragments by rill flow - a field study. Catena Suppl. 8: $35-54$.

PoESEn, J., Ingelmo-SÁNChez, F. \& MUCHER, H., 1990.- The hydrological response of soil surfaces to rainfall as affected by cover and position of rock fragments in the top layer. Earth Surface Processes and Landforms, vol.15.

RutZ-FlaÑo, P., GARCíA-RuIZ, J.M. \& ORTIGOSA, L., en prensa.- Geomorphological evolution of abandoned fields. A case study in the Central Pyrenees. Catena.

Ruiz-Flano, P., MARTínez-Rica, J.P. y GaRCiA-Ruiz, J.M., 1990.- Microambientes geomorfológicos en campos abandonados. Actas de la I Reunión Nacional de Geomorfologia. Teruel. 